\title{
Monte Carlo Capabilities of the SCALE Code System
}

\author{
B. T. Rearden ${ }^{1 *}$, L. M. Petrie ${ }^{1}$, D. E. Peplow ${ }^{1}$, K. B. Bekar ${ }^{1}$, D. Wiarda ${ }^{1}$, C. Celik ${ }^{1}$, C. M. Perfetti ${ }^{1}$, \\ A. M. Ibrahim ${ }^{1}$, S. W. D. Hart ${ }^{2}$, M. E. Dunn ${ }^{1}$, and W. J. Marshall ${ }^{1}$ \\ ${ }^{1}$ Oak Ridge National Laboratory, P.O. Box 2008, Oak Ridge, TN 37831, USA \\ *Corresponding Author, E-mail: reardenb@ornl.gov \\ ${ }^{2}$ University of Tennessee, Knoxville, TN 37921, USA
}

\begin{abstract}
SCALE is a widely used suite of tools for nuclear systems modeling and simulation that provides comprehensive, verified and validated, user-friendly capabilities for criticality safety, reactor physics, radiation shielding, and sensitivity and uncertainty analysis. For more than 30 years, regulators, licensees, and research institutions around the world have used SCALE for nuclear safety analysis and design. SCALE provides a "plug-and-play" framework that includes three deterministic and three Monte Carlo radiation transport solvers that can be selected based on the desired solution, including hybrid deterministic/Monte Carlo simulations. SCALE includes the latest nuclear data libraries for continuous-energy and multigroup radiation transport as well as activation, depletion, and decay calculations. SCALE's graphical user interfaces assist with accurate system modeling, visualization, and convenient access to desired results. SCALE 6.2, to be released in 2014, will provide several new capabilities and significant improvements in many existing features, especially with expanded continuous-energy Monte Carlo capabilities for criticality safety, shielding, depletion, and sensitivity and uncertainty analysis. An overview of the Monte Carlo capabilities of SCALE is provided here, with emphasis on new features for SCALE 6.2.
\end{abstract}

KEYWORDS: SCALE, KENO, MAVRIC, TSUNAMI, Monte Carlo, criticality safety, shielding, sensitivity analysis, depletion

\section{Introduction}

SCALE (Oak Ridge National Laboratory, 2011) is a widely used suite of tools, including recent nuclear data, for nuclear systems modeling and simulation that provides comprehensive, verified and validated, user-friendly capabilities for criticality safety, reactor physics, spent fuel and radioactive source term characterization, radiation shielding, and sensitivity and uncertainty analysis. SCALE is developed and maintained within the Reactor and Nuclear Systems Division of the Oak Ridge National Laboratory (ORNL). For more than 30 years, regulators, licensees, and research institutions around the world have used SCALE for nuclear safety analysis and design. SCALE provides a "plug-and-play" framework that includes three deterministic and three Monte Carlo radiation transport solvers that can be selected based on the desired solution, including hybrid deterministic/Monte Carlo methods for optimized performance. SCALE includes the latest nuclear data libraries for continuous-energy (CE) and multigroup (MG) radiation transport as well as activation, depletion, and decay calculations. All of SCALE's nuclear data are generated with the AMPX code system (Dunn and Greene, 2002), also developed at ORNL, for a truly independent methodology. SCALE's graphical user interfaces assist with accurate system modeling and visualization, and provide convenient access to the desired results. SCALE 6.2, to be released in 2014, will provide several new capabilities and significant improvements in many existing features, especially with expanded CE Monte Carlo capabilities for criticality safety, shielding, depletion, and sensitivity and uncertainty analysis.

SCALE provides separate Monte Carlo capabilities for eigenvalue neutronics and fixed-source coupled neutron-gamma calculations, in the KENO and Monaco codes, respectively (Goluoglu, et al, 2011; Peplow, 2011). Although the eigenvalue and fixed- source capabilities are provided in separate codes, there are many capabilities that are shared between them, including physics and geometry packages.

\section{Physics Treatment}

\subsection{Multigroup Physics}

The MG treatment implemented in SCALE has been in use since the 1960s and provides efficient and effective solutions with superior runtime performance. Problem-dependent MG cross-section data are temperature interpolated and resonance self-shielded by other SCALE modules prior to their use in each Monte Carlo 
calculation (Williams, 2011). Without proper resonance self-shielding, accurate MG calculations would not be possible for thermal or intermediate energy spectrum systems.

The multigroup cross sections consist of Bondarenko self-shielding factors, group length vectors of reaction cross sections, and two-dimensional Legendre expansions of energy transfer cross sections. After self-shielding has been accomplished and the two-dimensional expansions have been summed into a Legendre expansion of the total group-to-group transfer arrays, individual nuclide cross sections are multiplied by their densities and summed into mixtures. These mixture cross sections can then be used by the deterministic transport codes for their calculations. The Monte Carlo codes convert the Legendre expansion of the transfer arrays into probability distributions for the group-to-group transfers, and discrete scattering angles and probabilities that preserve the moments of the Legendre expansion of each group-to-group transfer. These transfer probabilities, angles, and angle probabilities are then transformed such that the new group and angle of scatter are efficiently selected through two random numbers, with only one multiplication and one addition operation. If the selected new group is negative, it is reset to positive and the new direction is chosen isotropically. If the problem is run with $\mathrm{P}_{1}$ scattering, the scattering angle is chosen from a continuous distribution. For higher order scattering, the polar scatter angle is discrete, and the azimuthal angle is randomly selected from a uniform distribution.

\subsection{Continuous-energy Physics}

The CE treatment in SCALE provides high-resolution solution strategies, with explicit physics representation. The CE data represent thermal scattering using free-gas and $s(\alpha, \beta)$, with explicit point-to-point data provided through the thermal region. The resolved resonance region is represented by pointwise data where the energy point density is optimized for each reaction of each nuclide. Data in the unresolved resonance region are represented by probability tables, and data above the unresolved region implement pointwise data with explicit point-to-point representation for secondary particles. CE physics contains non-transport data handling to support various flux, reaction rate, point detector tallies, and sensitivity analysis. In addition, CE data are converted from double-differential data format to lab format in a process where fast look-up tables are generated during the library generation. In SCALE 6.0-6.1, calculations are performed only at temperatures available on the data libraries by selecting the library temperature that is nearest to the desired temperature for the calculation.

\section{Geometry Packages}

There are two variants of KENO that provide identical solution capabilities with different geometry packages. KENO V.a implements a simple and efficient geometry package that is sufficient for modeling many systems of interest to criticality safety and reactor physics analysts. KENO-VI implements the SCALE Generalized Geometry Package (SGGP), which provides a quadratic-based geometry system with much greater flexibility in solution modeling. Monaco implements only the SGGP geometry package. Both packages are based on solid bodies that are organized into reusable objects called units that are constructed of material regions. Units can be conveniently arranged in rectangular or hexagonal arrays of repeating units. Additionally, nesting is available such that one unit can contain another unit as a hole or an array can be nested inside of a unit, which itself can be repeated in another array. There is no limit to the number of nesting levels available, so very complex systems can be quickly generated.

\subsection{KENO V.a Geometry}

KENO V.a models are constructed from regions of specific shapes following strict rules, which provide great efficiency in geometry tracking. Allowed shapes are cubes, cuboids (rectangular parallelepipeds), spheres, cylinders, hemispheres, and hemicylinders. These shapes must be oriented along orthogonal axes and can be translated but cannot be rotated. A major restriction applied to KENO V.a geometry is that intersections are not allowed, and each region of a unit must fully enclose the preceding region. KENO V.a provides rectangular arrays, where the outer body of each unit contained in the array must have cuboidal shape and adjacent faces must have the same dimensions. The entire array must be fully enclosed by the region in which it is placed. An example KENO V.a model of a spent fuel transportation package is shown in Figure 1. 


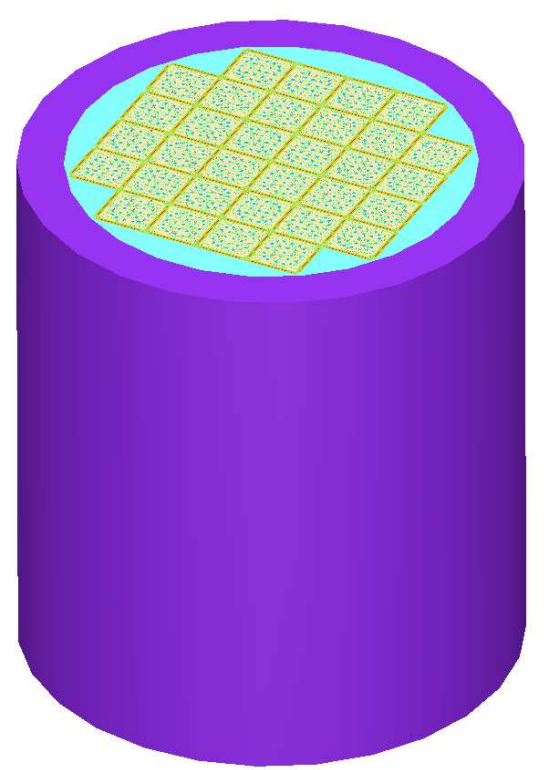

Figure 1: KENO V.a model of a spent fuel transportation package.

\subsection{SGGP Geometry}

SGGP is a quadratic-based geometry system that provides predefined bodies including cone, cuboid, cylinder, dodecahedron, ecylinder (elliptical cylinders), ellipsoid, hexprism, hopper (truncated pyramid), parallelepiped, planes, rhombohedron, rhexprism (rotated hexprisms), sphere, and wedge. Additionally, bodies not directly provided with SGGP can be constructed from quadratic surfaces defined with user-input coefficients. All bodies and surfaces can be rotated and translated to any orientation and position within their respective unit. SGGP also provides intersecting regions.

SGGP arrays may be composed of cuboids, hexprisms, rhexprisms, or dodecahedrons. Like KENO V.a, the faces of adjacent units in an array must have the same dimensions. An array boundary must be specified for each array, and only that portion of the array within the boundary is considered a part of the system. Also, the specified array must fill the entire volume in the specified array boundary. The array boundary may be any shape that can be specified using quadratic equations.

The use of holes is more flexible in SGGP than in KENO V.a. Within a unit, holes cannot intersect other holes or the unit boundary, but they can intersect region boundaries. The use of holes is not necessary to build complex geometries; they are used primarily to more efficiently build complex geometries and improve the tracking efficiency of the simulation. In SGGP the distance to each surface in the unit must be calculated after each collision. By moving some of the surfaces in a unit into another unit that is included as a hole, all the surfaces in the hole unit except the outer boundary are removed from the containing unit. The judicious use of holes in SGGP can significantly speed up the calculation. An example KENO-VI model of a transportation package with hexagonally pitched fuel arrays is shown in Figure 2. 


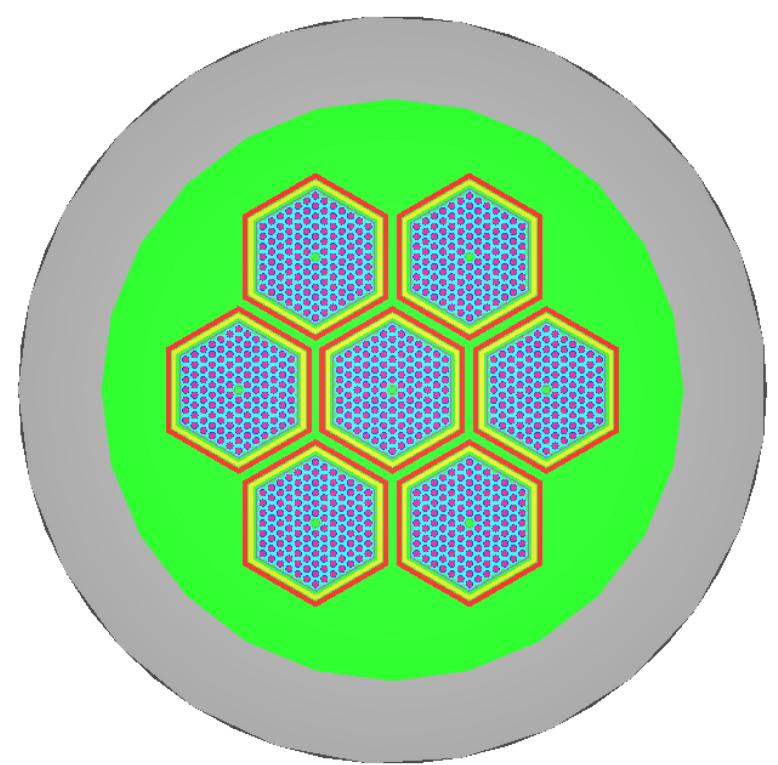

Figure 2: KENO-VI model of the CASTOR shipping cask.

\section{Eigenvalue Analysis}

KENO V.a and KENO-VI perform eigenvalue calculations for neutron transport primarily to calculate multiplication factors and flux distributions of fissile systems in both CE and MG mode. Both codes allow explicit geometric representation with their respective geometry packages. KENO provides a MG adjoint capability, which is especially useful for sensitivity analysis. KENO implements standard variance reduction techniques such as implicit capture, splitting, and Russian roulette.

The initial fission source distribution in KENO can be specified with nine options. These options include the default option of a uniform distribution throughout the fissile material; an axially varying distribution input by the user or defined as $\cos (\mathrm{Z})$ or $(1-\cos (\mathrm{Z}))^{2}$, where $\mathrm{Z}$ is the axial position; several options to initialize the source at a given position, within a given volume, within a given unit, or within a unit at a specified array index; or to specifically provide the coordinates of each starting point.

KENO approximates the real $k_{\text {eff }}$ variance using an iterative approach and lagging covariance data between generations (Ueki, 1997). KENO provides a $\chi^{2}$ test for the normality of $k_{\text {eff }}$ and provides plots of $k_{\text {eff }}$ by active and inactive generations. KENO reports a best estimate of $k_{\text {eff }}$ that is computed as the minimum variance of $k_{\text {eff }}$ based on generations skipped and generations run.

KENO provides track-length tallies for scalar flux and angular flux moments needed for sensitivity analysis. Additionally, tallies are provided for reaction rates, with isotopic tallies available only in CE calculations. KENO also provides mesh tallies based on a user-input orthogonal grid.

Matrix $k_{\text {eff }}$ calculations provide an additional method of calculating the $k_{\text {eff }}$ of the system. Cofactor $k_{\text {eff }}$ and source vectors, which describe the contribution to the system $k_{\text {eff }}$ from each unit, hole, or array, are available.

\section{KENO Enhancements for SCALE 6.2}

KENO has been substantially improved for SCALE 6.2, especially for CE calculations, source convergence diagnostics and acceleration, and parallel capabilities.

\subsection{Reduction in Memory Requirement of $\mathrm{CE}$ Internal Storage}

Continuous-energy calculations in SCALE 6.0-6.1 have been performed using a unionized energy grid, where material-dependent cross-section data are generated for each user-defined mixture on a uniform grid as the calculation begins. For an example uranyl nitrate solution, a smooth cross section like ${ }^{1} \mathrm{H}$ that generally has very few energy points must be refined to the same energy resolution as ${ }^{238} \mathrm{U}$, which has over 50,000 energy points. Additionally, the unionized energy grid results in substantially increased memory usage for a problem with many materials, because each cross section is fully duplicated in memory for each material where it is used. For 
example, a model with spent nuclear fuel with 18 axial zones would require 18 copies of each cross section on the unionized grid, one for each use of the same data in the model. With this data format, even basic criticality safety models with impurities modeled could require many gigabytes of memory, and applications problems with multiple spent fuel mixtures could easily exceed 100 GB of memory, making the tool of limited practical value.

To mitigate the problems with previous implementations, an interpolated cross-section calculation capability has been introduced as an option in KENO to disable the use of the unionized energy grid for all materials in a mixture. KENO calculations with this new option provide a dramatic reduction in memory requirements that can be more than an order of magnitude, depending on the materials used in the model, with a runtime penalty of a few percent.

Internal storage of CE cross-section data has been converted from double precision to single precision, which results in a further $15-45 \%$ reduction in memory footprint, depending on the problem, with no loss of precision in computed results. With these and other refinements in data storage and use strategies, models with many regions of spent fuel can now be modeled with approximately 2 GB of memory.

\subsection{Fission Source Convergence Diagnostics}

Prior to SCALE 6.2, KENO provided only plots of $k_{\text {eff }}$ by generation and average $k_{\text {eff }}$ for visual inspection of source convergence, followed by a $\chi^{2}$ statistical assessment of convergence. With SCALE 6.2, fission source convergence diagnostic techniques have been implemented in KENO to provide improved confidence in the computed results as well reduce in the simulation time for some cases. Confirming the convergence of the fission source distribution is especially useful to avoid the false convergence of $k_{\text {eff }}$ that can be caused by insufficient sampling of important portions of the system (Ueki and Brown, 2005).

KENO source convergence diagnostics rely on Shannon entropy statistics of the mesh-based fission source data. In order to accumulate the fission source on a grid, multiple mesh support has been added to KENO. Formerly, KENO supported only a single mesh definition for either mesh-flux calculations for use in sensitivity and uncertainty analysis or for fission source accumulations for use in criticality alarm system analysis. The multiple-mesh capability enables accumulating these quantities on different grids and also enables the accumulation of fission source data for convergence diagnostics. Figure 3 illustrates convergence of the eigenvalue and fission source distribution for a challenging source convergence benchmark problem (Blomquist, et al, 2006). These results confirm that evaluating eigenvalue results with Shannon entropy avoids getting false-converged $k_{\text {eff }}$ values. KENO source convergence diagnostics indicate that the fission source becomes stationary in 5,018 generations for this model problem.

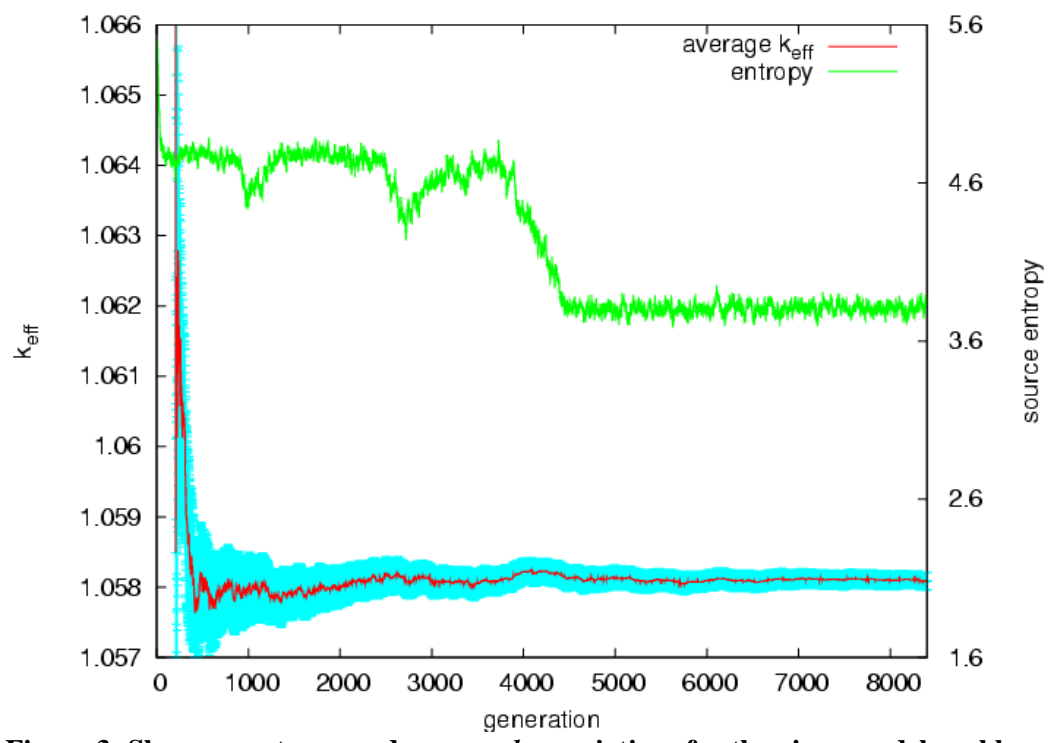

Figure 3: Shannon entropy and average $\boldsymbol{k}_{\text {eff }}$ variations for the given model problem.

\subsection{Doppler Broadened Rejection Correction}

The implementation of Doppler Broadened Rejection Correction (DBRC) techniques provides further enhancements for calculations with elevated temperatures (Hart, 2013). As shown in Table 1, DBRC in KENO 
presents a reactivity correction of approximately $300 \mathrm{pcm}$ relative to the default methodology for a $1200 \mathrm{~K}$ light water reactor (LWR) fuel pin, consistent with that predicted with MCNPX by the originators of the methodology (Becker, et al, 2009).

\begin{tabular}{cccc}
\hline Case & Default & DBRC & $\begin{array}{c}\text { Difference } \\
(\mathrm{pcm})\end{array}$ \\
\hline MCNPX & $1.31137+/-9 \mathrm{E}-5$ & $1.30791+/-9 \mathrm{E}-5$ & -346 \\
\hline KENO VI & $1.31029+/-15 \mathrm{E}-5$ & $1.30730+/-15 \mathrm{E}-5$ & -299 \\
\hline
\end{tabular}

Table 1: Effect of DBRC for a $L W R$ fuel pin at 1200K

\subsection{Problem-Dependent Doppler Broadening}

The CE data libraries distributed with SCALE are provided with only about five temperatures per isotope. The Doppler broadening temperature corrections using only a few temperatures may not match the desired temperature of the calculation. When temperatures of the KENO model are different from those present on the library, KENO selects the nearest temperature, which can be several hundred degrees from the desired temperature, producing results that can vary significantly from those that would be produced at the correct temperature.

A finite difference method has been implemented to provide problem-dependent temperature corrections by Doppler broadening the pointwise data in the resolved resonance region and the probability tables in the unresolved resonance region when the cross sections are loaded for the calculation (Larson, 2008). The eigenvalues computed for a typical fresh pressurized water reactor (PWR) pin cell using the interpolated MG temperature correction, nearest selected $\mathrm{CE}$ temperature, and problem-dependent $\mathrm{CE}$ temperature treatments are shown in Figure 4. In addition to this ongoing effort, there are plans to determine ways to broaden the the $S(\alpha, \beta)$ data as well.

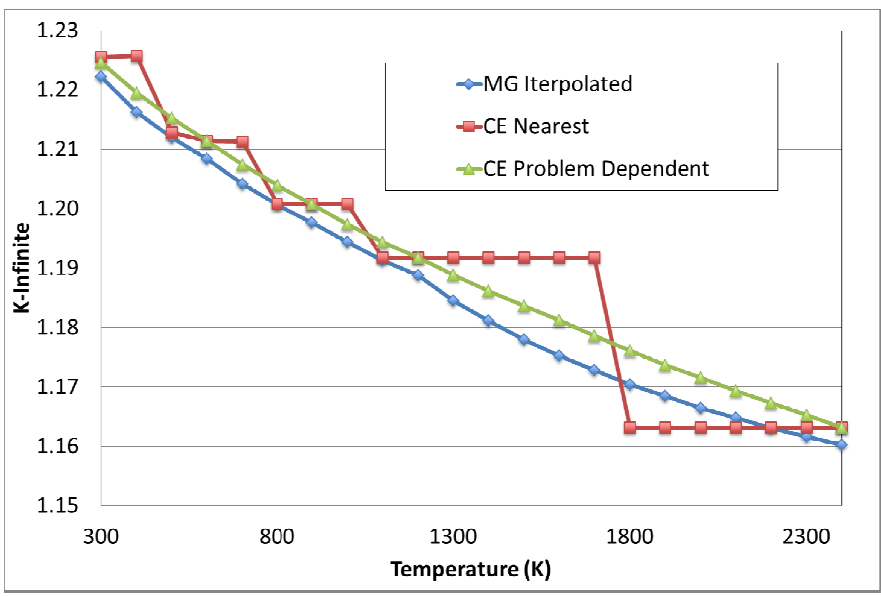

Figure 4: Eigenvalues computed for a PWR pin cell with different temperature treatments.

\subsection{Hybrid Method for Starting Source Distribution}

The Sourcerer sequence introduced in SCALE 6.2 uses the solution from the Denovo (Evans, 2010) discrete-ordinates code (through the DEVC sequence) as that starting fission source distribution in a KENO Monte Carlo calculation. Initial studies (Evans, 2010; Ibrahim, et al, 2012) have shown that using a starting fission distribution that is similar to the true fission distribution can both reduce the number of skipped generations required for fission source convergence and significantly improve the reliability of the final $k_{e f f}$ result.

For many criticality safety applications, the additional step of performing a deterministic calculation to initialize the starting fission source distribution is not necessary. However, for challenging criticality safety analyses, such as as-loaded spent nuclear fuel transportation packages with a mixed loading of low- and high-burnup fuel, even a low-fidelity deterministic solution for the fission source produces more reliable results than the typical starting distributions of uniform or cosine functions over the fissionable regions, as demonstrated in a recent study (Ibrahim, et al, 2013). In that study, a cask holding 24 spent fuel assemblies was examined using a uniformly distributed starting source and a deterministically calculated starting source. Multiple clones of KENO were run (with different random number seeds) for different values of skipped cycles. The number of 
clones that gave an incorrect result for $k_{\text {eff }}$ was then tabulated. The results from that study, presented in Figure 5, show that using a deterministic starting source significantly increases the $k_{\text {eff }}$ reliability.

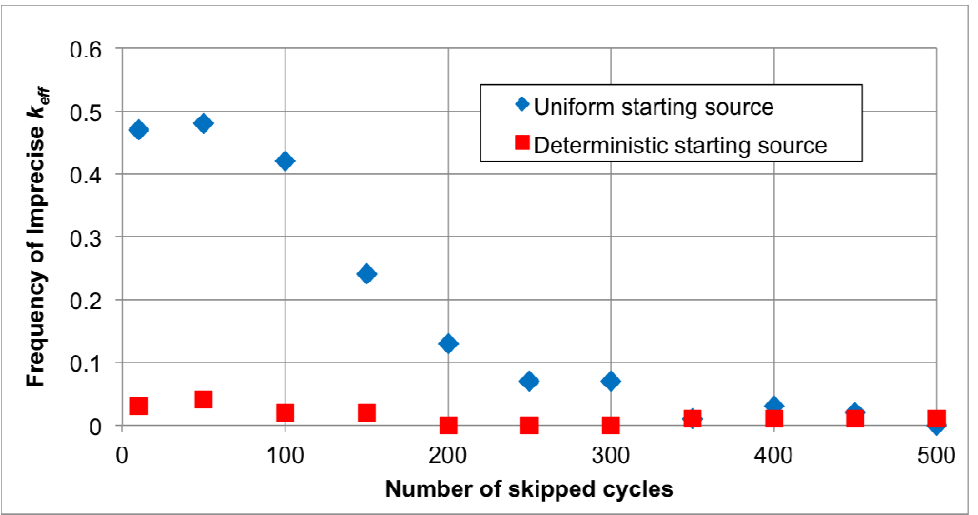

Figure 5: Fraction of failure to agree with the reference $k_{\text {eff }}$ value for KENO calculations with different starting sources.

\subsection{Distributed Memory Parallelism via MPI}

In addition to the numerous improvements described that directly affect solution accuracy, parallel computation capabilities have been added to KENO to provide reductions in wall clock time, especially for S/U analysis or Monte Carlo depletion on computer clusters. By introducing a simple master-slave approach via Message Passing Interface (MPI) (Gabriel, et al, 2004), KENO runs different random walks concurrently on the replicated geometry within the same generation. The fission source and other tallied quantities are gathered at the end of each generation by the master process; then, they are processed either for final edits or next generations.

The parallel performance of KENO as used in a CE calculation for a graphite-moderated reactor model is shown in Figure 6. Here, it can be observed that speedup increases very rapidly as a function of the number of processors. The parallel performance of KENO is very good up to eight processors (greater than 93\%). The parallel performance starts diminishing as a function of increased number of processors but is still above $80 \%$ when using up to 20 processors.

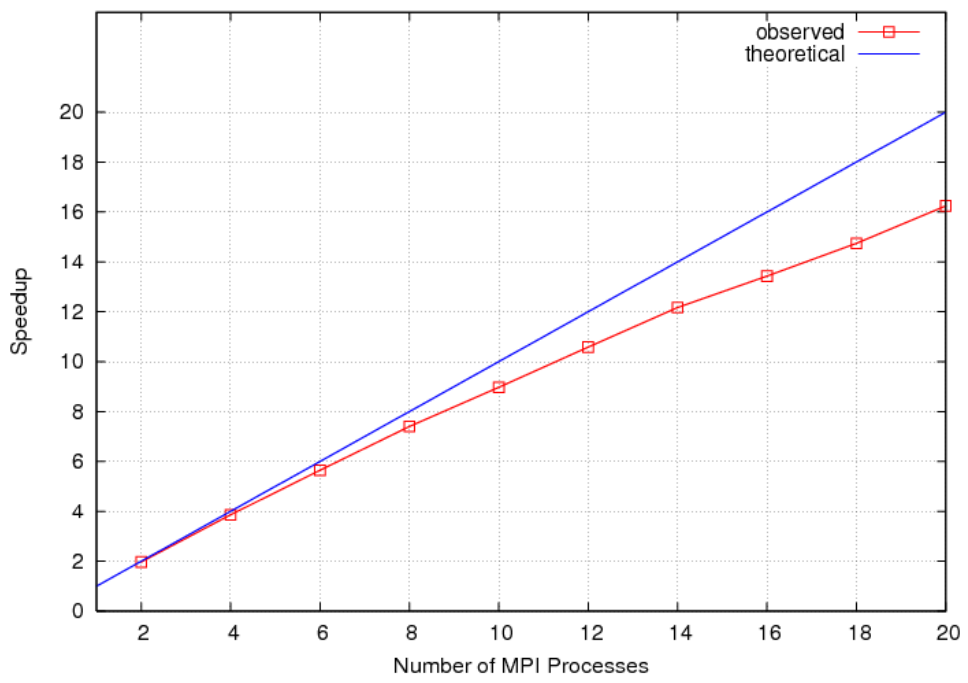

Figure 6: Speedup for parallel KENO-VI calculations for a graphite-moderated reactor model.

\section{Shielding Analysis}

Monaco is a fixed-source Monte Carlo shielding code that calculates neutron and photon fluxes and response functions for specific geometry regions, point detectors, and mesh tallies (Goluoglu, et al, 2011). In addition, Monaco has variance reduction capabilities, such as source biasing and weight windows, which can be 
automated via the MAVRIC sequence (Monaco with Automated Variance Reduction using Importance Calculations). MAVRIC performs radiation transport on problems that are too challenging for standard, unbiased Monte Carlo methods. The intention of the sequence is to calculate fluxes and dose rates with low uncertainties in reasonable times even for deep penetration problems. MAVRIC automatically performs a coarse mesh, three-dimensional, discrete-ordinates calculation using Denovo to determine the adjoint flux as a function of position and energy. This adjoint flux information is then used by MAVRIC to construct a space and energy-dependent importance map (i.e., weight windows) to be used for biasing during particle transport and a mesh-based biased source distribution. MAVRIC then passes the importance map and biased source distribution to Monaco.

MAVRIC uses an adjoint deterministic calculation to construct biasing parameters to optimize a single tally or can use both forward and adjoint deterministic calculations to construct biasing parameters to optimize multiple tallies or a mesh tally. For example, when computing fluxes in the reactor vessel of the Monte Carlo Performance Benchmark problem (Hoogenboom, et al, 2011), long times are needed to converge the values at the outer edge. Fluxes at the center of the reactor converge quickly; however, neutrons rarely penetrate the entire vessel. With MAVRIC's mesh tally optimization, source particles are sampled more frequently at the edge of the core and the importance map prevents the neutrons from spending too much time at the core center, producing more uniform convergence in the high- and low-flux areas. Figure 7 shows the relative uncertainty in the flux mesh tally for a 10 hour analog calculation, and Figure 10 shows the relative uncertainty for the globally optimized calculation using a 17-minute forward deterministic, a 31-minute adjoint, and a 558-minute MC calculation (total time of 606 minutes). The analog calculation has uncertainties greater than 10\% for most of the vessel, while the center of the core has less than $1 \%$. The globally optimized calculation has $1-2 \%$ relative uncertainties in most voxels. Using the average relative uncertainty over the entire mesh tally as the metric, the calculation with the biased source and importance map has a figure of merit that is 277 times higher than the analog calculation.

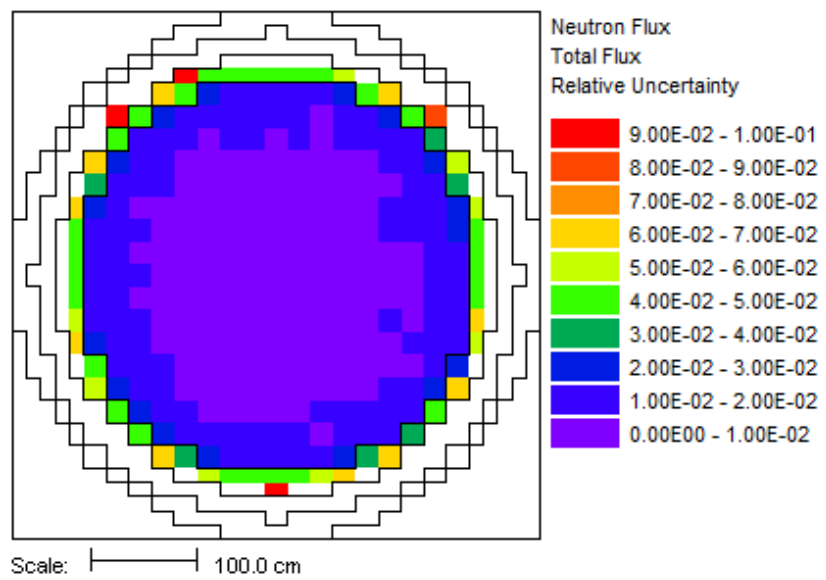

Figure 7: Relative uncertainties in total flux for the center plane of the MCPB problem using analog MC. Values above $10 \%$ are not shown. 


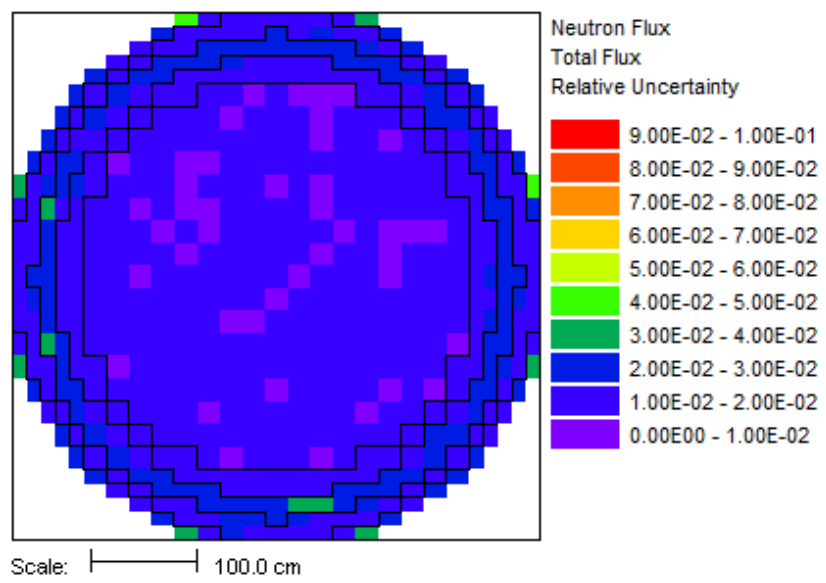

Figure 8: Relative uncertainties in total flux for the center plane of the MCPB problem using global optimization.

\section{Shielding Enhancements for SCALE 6.2}

Prior to SCALE 6.2, the MAVRIC/Monaco capabilities relied on the MG approach for radiation transport. The MG approach is suitable for many applications but can be problematic for others. For example, deep penetration shielding through iron, where the MG-averaged cross section for capture resonances may not accurately represent the true transmission of neutrons due to fine "windows" through (i.e., minima in) the cross section. Additionally, MG cannot adequately resolve discrete gamma emission lines, such as those of ${ }^{60} \mathrm{Co}$. With the CE treatment introduced in SCALE 6.2, improved solution fidelity is available. The generation of CE nuclear data and the implementation of CE physics in Monaco are based on a first-principles approach, where the simulation is represented as realistically as possible. This approach will lead to higher-fidelity results, but runtimes may be substantially increased over more approximate methods.

\subsection{SCALE CE Modular Physics Package}

A new package was developed to serve as the CE collision physics engine, called SCALE Continuous-Energy Modular Physics Package (SCEMPP). SCEMPP models particle collisions in a material and generates the particle(s) resulting from a collision. Essentially, SCEMPP is an event generator for SCALE. SCEMPP has Fortran and $\mathrm{C}++$ application programming interfaces (APIs) in order to support both legacy and future developments in SCALE. SCEMPP requires cross sections and kinematics data for each isotope/element and has only one dependency to the CE Resource package, which is another new feature in the SCALE code development to read and store all the nuclear data from AMPX CE particle libraries and transfer information via APIs. Communication through APIs, unlike traditional I/O operations in SCALE, and SCEMMP's generic modular structure enable a flexible and yet powerful integration of capabilities within the code modernization development in SCALE. In addition to creating collision particles, SCEMPP also provides non-transport data, such as reaction responses or point detector data, to Monaco in order to enable dose calculations and point detector tally estimates.

\subsection{CE MAVRIC/Monaco}

CE calculations in Monaco use SCEMPP for particle transport, and the currently available features in Monaco have been improved to enable MG and CE calculations with a consistent and user-friendly input. CE Monaco can operate in neutron, photon, and neutron-photon coupled particle transport modes. All the available tally types in Monaco have been improved to use user-defined energy structures and particle type (neutron or photon). MAVRIC always requires a MG library to enable variance reduction calculations with the Denovo $S_{n}$ code. However, the final Monaco calculation in the MAVRIC sequence can now be performed with either MG or CE physics.

One area where the impact of MG is detrimental is the transport of mono-energetic photons. In MG, all photons within a group use the same averaged cross section. Consider a ${ }^{60}$ Co source and a tungsten shield. The cross sections for the two ${ }^{60} \mathrm{Co}$ line sources are listed in Table 2 for the different cross-section libraries. The small differences in cross section can make large differences in the transport. Consider just $5 \mathrm{~cm}$ of tungsten. Using the cross sections in Table 2 , the attenuation $\left(e^{-\mu x}\right)$ of either line can vary by $30 \%$. 


\begin{tabular}{l|cc}
\hline & $1.173230 \mathrm{MeV}$ & $1.332490 \mathrm{MeV}$ \\
\hline SCALE CE & 1.03353 & 0.94864 \\
SCALE 47-grp & 1.09066 & 0.92743 \\
SCALE 19-grp & 1.05167 & 0.89289 \\
\hline
\end{tabular}

Table 2: Total macroscopic cross section in tungsten $(/ \mathrm{cm})$.

For line photon sources in deep penetration shielding problems, the difference in fluxes and dose rates between MG and CE can be quite large. Consider the AOS-100 radioactive transport package consisting of steel and tungsten $(71.12 \mathrm{~cm}$ outer diameter and $91.44 \mathrm{~cm}$ height). Predicted dose rates outside the package due to a 1 $\mathrm{Ci}{ }^{60} \mathrm{Co}$ source in the inner cavity $(16.51 \mathrm{~cm}$ diameter and $50.8 \mathrm{~cm}$ height) using the 19 -group cross sections can be 2-3 times higher than the CE predictions, as shown in Figure 9. Dose rates with the 47-group cross sections are closer to $\mathrm{CE}$ but can still be about $50 \%$ higher than the CE dose rates.
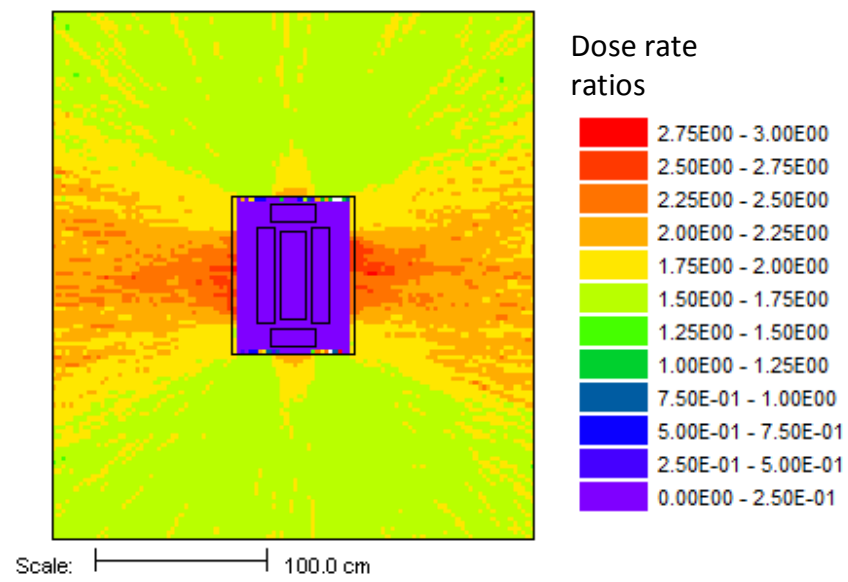

Figure 9: Simple AOS-100 package geometry and ratio of the 19-group MG computed dose rates to the CE dose rates.

\section{Sensitivity and Uncertainty Analysis}

\subsection{MG Eigenvalue Sensitivity Analysis}

Sensitivity coefficients describe the fractional change in a system response that is induced by changes to system parameters and nuclear data. The Tools for Sensitivity and UNcertainty Analysis Methodology Implementation (TSUNAMI) code within the SCALE code system makes use of eigenvalue sensitivity coefficients for an extensive number of criticality safety applications, such as quantifying the data-induced uncertainty in the eigenvalue of critical systems, assessing the neutronic similarity between different critical systems, and guiding nuclear data adjustment studies (Rearden, et al, 2011).

TSUNAMI facilitates the application of sensitivity and uncertainty theory to criticality safety analysis using 3D Monte Carlo models by performing all necessary steps to compute sensitivity coefficients from a single input file. Data computed with TSUNAMI are the sensitivities of $k_{\text {eff }}$ to each constituent cross-section data component used in the calculation. TSUNAMI also provides automated, problem-dependent resonance self-shielded cross-section data using the same methods and input as the SCALE criticality safety sequences. However, to provide an accurate estimation of the sensitivity coefficients for systems in which resonance self-shielding is important, the sensitivity coefficients require additional terms to account for the first-order implicit effect of perturbations in the material number densities or nuclear data upon the shielded group-wise macroscopic cross-section data (Williams, et al, 2001). For example, in a water-moderated, low-enriched-uranium system, the resonance self-shielded cross section for ${ }^{238} \mathrm{U}(\mathrm{n}, \gamma)$ is dependent on the moderation of neutrons by ${ }^{1} \mathrm{H}$. Thus, the sensitivity of $k_{\text {eff }}$ to ${ }^{1} \mathrm{H}$ elastic scattering has an implicit component introduced by its influence on the resonance self-shielded cross section for ${ }^{238} \mathrm{U}(\mathrm{n}, \gamma)$, which leads to a change in $k_{\text {eff }}$ for the system. This implicit effect can account for several percent of the overall sensitivity of $k_{e f f}$ to the cross-section data and often requires additional consideration in the model setup.

After the cross sections are processed, MG TSUNAMI performs two KENO criticality calculations, one forward and one adjoint, to compute the energy and spatially dependent flux solutions and their angular moments 
(Rearden, 2004). The user must provide adequately fine spatial resolution of the flux solutions to allow for appropriate folding of the forward and adjoint solutions. This is accomplished either by manually entering geometry divisions or by using the automated meshing features of KENO developed specifically for this purpose. TSUNAMI provides for separate control of the forward and adjoint calculations to independently specify the number of particles per generation, the number of generations, the number of generations skipped before accumulating data, and the desired convergence criteria for each calculation.

The analyst's selection of modeling strategies and TSUNAMI input parameters can significantly affect the sensitivity profiles generated by TSUNAMI. Erroneous implicit sensitivity coefficients may result if the cross-section resonance self-shielding model is inconsistent with the use of the material in the KENO model. Thus, the importance of performing a thorough set of direct perturbation calculations to verify the accuracy of the TSUNAMI sensitivity data cannot be overemphasized.

Finally, TSUNAMI calls SAMS, where the flux moment product terms are computed and the sensitivity coefficients are computed. SAMS also computes the uncertainty in the sensitivity coefficients introduced by uncertainties in the Monte Carlo calculations. SAMS prints energy-integrated sensitivity coefficients and their uncertainties to the SCALE output file and generates a sensitivity data file (SDF) containing the energy-dependent sensitivity coefficients and their uncertainties for use in subsequent analysis and validation activities.

\subsection{CE Eigenvalue Sensitivity Analysis}

With SCALE 6.2, the MG eigenvalue sensitivity and uncertainty analysis methods that use KENO for transport analysis are extended to provide CE capabilities through the implementation of the CLUTCH (Contributon-Linked eigenvalue sensitivity/Uncertainty estimation via Tracklength importance CHaracterization) methodology and IFP (Iterated Fission Probability) methods (Perfetti and Rearden, 2013). CLUTCH is an efficient methodology that has been demonstrated to provide high-fidelity results with manageable run-times and memory requirements. State-of-the-art sensitivity methods make CE calculations easier to learn and use than MG in several ways; for example, CE calculations do not require resonance self-shielding calculations to determine implicit sensitivity effects, the simulation of a separate adjoint transport calculation, or the use of a flux mesh for tallying fluxes and flux moments. Furthermore, CE calculations provide the capability to tally sensitivities with very fine energy resolution, as shown in Figure 10.

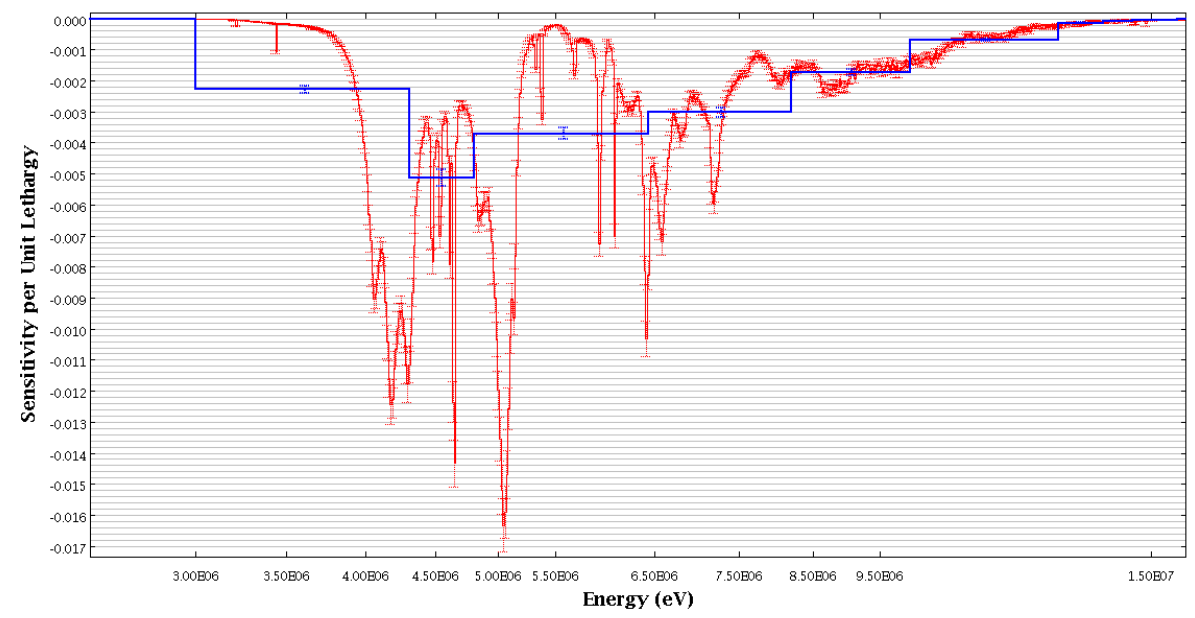

Figure 10: Sensitivity of $k_{\text {eff }}$ to ${ }^{16} \mathrm{O}$ capture computed with MG TSUNAMI in 238 groups (blue) and CE TSUNAMI binned in 10,000 groups (red).

The move to CE physics allows for improvements in sensitivity coefficient accuracy compared to MG. Table 3 compares the accuracy of the total nuclide sensitivity coefficients estimated by the each sensitivity method for the MIX-COMP-THERM-004-001 system from the International Handbook of Evaluated Criticality Safety Benchmark Experiments (IHECSBE) (for Economic Cooperation and Development, 2012). The MG analysis produced a ${ }^{238} \mathrm{U}$ total nuclide sensitivity that disagreed with the reference direct perturbation sensitivity by 2.80 effective standard deviations $\left(\sigma_{e f f}\right)$, while the CE methods (IFP and CLUTCH) sensitivities were within almost 1 standard deviation of the reference sensitivity coefficients for all of the nuclides examined. 
Figure 11 compares the figures of merit for the nuclide sensitivity coefficients presented in Table 3 . Figure 11 indicates that the CE sensitivity calculations are of a comparable efficiency to the MG calculations, and in some cases the CE CLUTCH method is more efficient than MG method. This is remarkable because of the use of CE physics, which can impose relatively long cross-section lookup times, and indicates the efficiency of the CLUTCH method.

\begin{tabular}{ccccc}
\hline \multirow{2}{*}{ Sensitivity } & Reference & MG TSUNAMI & \multicolumn{2}{c}{ CE TSUNAMI } \\
& & & IFP & CLUTCH \\
\hline \multirow{2}{*}{$\mathrm{H}_{2} \mathrm{O}$} & $0.2935 \pm$ & $0.2805 \pm$ & $0.2733 \pm$ & $0.2793 \pm$ \\
& 0.0179 & 0.0088 & 0.0052 & 0.0065 \\
& & $\left(-0.65 \sigma_{\text {eff }}\right)$ & $\left(-1.08 \sigma_{\text {eff }}\right)$ & $\left(-0.75 \sigma_{\text {eff }}\right)$ \\
& & $-0.0050 \pm$ & $-0.0055 \pm$ & $-0.0057 \pm$ \\
${ }^{238} \mathrm{U}$ & $-0.0061 \pm$ & 0.0002 & 0.0003 & 0.0001 \\
& 0.0003 & $\left(2.80 \sigma_{\text {eff }}\right)$ & $\left(1.16 \sigma_{\text {eff }}\right)$ & $\left(1.14 \sigma_{\text {eff }}\right)$ \\
& & $0.1264 \pm$ & $0.1188 \pm$ & $0.1190 \pm$ \\
${ }^{239} \mathrm{Pu}$ & $0.1262 \pm$ & 0.0014 & 0.0020 & 0.0001 \\
& 0.0087 & $\left(0.02 \sigma_{\text {eff }}\right)$ & $\left(-0.83 \sigma_{\text {eff }}\right)$ & $\left(-0.83 \sigma_{e f f}\right)$ \\
& & $-0.03750 \pm$ & $-0.03738 \pm$ & $-0.03743 \pm$ \\
${ }^{240} \mathrm{Pu}$ & $-0.03777 \pm$ & 0.00011 & 0.00060 & 0.00002 \\
& 0.00350 & $\left(0.08 \sigma_{\text {eff }}\right)$ & $\left(0.11 \sigma_{\text {eff }}\right)$ & $\left(0.10 \sigma_{\text {eff }}\right)$ \\
& & $0.00599 \pm$ & $0.00567 \pm$ & $0.00579 \pm$ \\
& $0.00589 \pm$ & 0.00004 & 0.00014 & 0.00003 \\
${ }^{241} \mathrm{Pu}$ & 0.00042 & $\left(0.24 \sigma_{\text {eff }}\right)$ & $\left(-0.50 \sigma_{\text {eff }}\right)$ & $\left(-0.24 \sigma_{\text {eff }}\right)$ \\
\hline
\end{tabular}

Table 3: MIX-COMP-THERM-004-001 total nuclide sensitivity coefficient comparison.

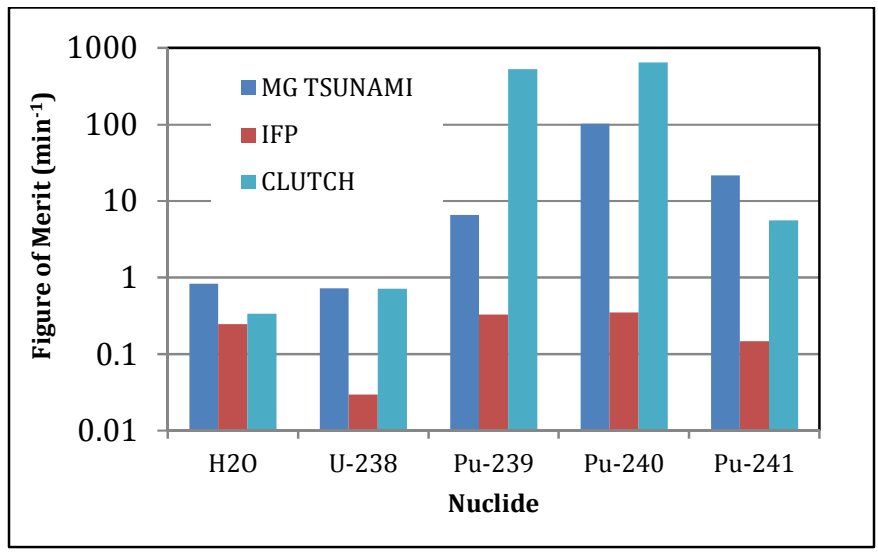

Figure 11: MIX-COMP-THERM-004-001 total nuclide sensitivity Figure of Merit comparison.

Table 4 gives the amount of computational memory required by each method for the sensitivity coefficient calculations. These memory requirements were obtained by subtracting the memory requirements of each eigenvalue-only calculation from the memory requirements of each sensitivity coefficient calculation. As seen below, the CLUTCH method excels in terms of memory usage, and can readily compute sensitivities for complex systems on a typical personal computer with 8 GB of RAM.

\begin{tabular}{cccc}
\hline \multirow{2}{*}{ Model } & \multirow{2}{*}{ MG } & \multicolumn{2}{c}{ CE TSUNAMI } \\
\cline { 3 - 4 } & TSUNAMI & IFP & CLUTCH \\
\hline MIX-COMP-THERM-004-001 & $13,785 \mathrm{MB}$ & $10,643 \mathrm{MB}$ & $63 \mathrm{MB}$ \\
\hline
\end{tabular}

Table 4: Sensitivity method memory usage. 


\subsection{Sampler}

A new stochastic uncertainty quantification capability has been added with the SCALE 6.2 Sampler module that implements stochastic techniques to quantify the uncertainty in any computed result from any SCALE sequence due to uncertainties in

- neutron cross sections,

- fission yield and decay data, and

- any user input parameter, such as geometry, material density, isotopic composition, temperature, etc.

Sampler propagates these uncertainties through complex analysis sequences, such as depletion calculations, and provides the variation in the output quantities due to variations in any combination of input data (Williams, et al, 2013). Correlations between systems with shared uncertainties are also computed, which is especially useful for quantifying correlated uncertainties in benchmark experiments, required for generalized linear least-squares techniques implemented by the SCALE module TSURFER (Rearden, et al, 2013).

\section{Monte Carlo Depletion}

SCALE 6.1 provided MG Monte Carlo depletion that coupled SCALE MG cross-section processing capabilities with KENO and ORIGEN (Oak Ridge Isotope GENeration) via the TRITON sequence (DeHart and Bowman, 2011). In addition to the existing MG capability, a new CE-based KENO/ORIGEN Monte Carlo depletion capability has been developed for SCALE 6.2 and can be utilized by simply changing the input library specification (Goluoglu, et al, 2012). CE depletion is especially useful for models with complex geometry that present difficulties in obtaining accurate resonance self-shielded MG data and for models with many depletion regions where run-time to generate and store the resonance self-shielded cross-section data for each material is prohibitive.

TRITON performs CE depletion calculations using ORIGEN to update region-wise nuclide inventories at each burnup step by integrating cross-section data with the flux spectrum for each depletion region. In addition to the reaction cross-section data utilized in the Monte Carlo calculation, energy-dependent fission product yield data and additional activation cross sections must be integrated with the scalar flux at each depletion step. In MG mode, resonance self-shielded cross-section data are generated prior to the KENO calculation. SCALE provides MG Monte Carlo depletion by integrating the scalar flux with the resonance self-shielded multigroup cross-section data with a utility module outside of the KENO Monte Carlo calculation. The predictor-corrector methodology is implemented for depletion.

In some cases, MG depletion suffers from the inadequacies of the MG approximations such as inadequate group structure or inability to properly shield the cross sections for the problem using the one-dimensional resonance self-shielding modules available in SCALE. Additionally, the requirement to update the resonance self-shielding at each depletion step can present a large computational burden that makes calculations impractical or impossible because the storage of independent sets of MG data for thousands of depletion materials can require excessive amounts of memory. To alleviate issues associated with the MG technique while maintaining components of the current depletion infrastructure of SCALE, a few-group microscopic reaction cross-section calculation capability is now available for CE calculations in KENO.

Results for a benchmark based on destructive isotopic assay data for sample MKP109-P of assembly D047 irradiated in the Calvert Cliffs PWR to a burnup of $44 \mathrm{GWd} / \mathrm{MTU}$ are shown below in Table 5 (Ilas, et al, 2010). Here the percent differences from the calculated (C) to experimental (E) values are shown to be similar between MG and CE calculations on the same KENO models. However, for CE depletion, it is not necessary to compute and track resonance self-shielded cross sections for each depletion region separately. For models with hundreds or thousands of depletion regions, the previously described advancements in CE calculations provide substantially reduced memory requirements, enabling high-fidelity calculations that were not previously possible. 


\begin{tabular}{|c|c|c|c|c|c|}
\hline & $\mathrm{MG}$ & $\mathrm{CE}$ & & $\mathrm{MG}$ & $\mathrm{CE}$ \\
\hline U-234 & 3.0 & 3.2 & Am-241 & -8.2 & -5.8 \\
\hline U-235 & 3.1 & 4.6 & Cs-133 & -0.8 & 0.7 \\
\hline U-236 & 0.1 & 1.4 & Cs-135 & 3.0 & 4.2 \\
\hline U-238 & -1.7 & -0.1 & Cs-137 & -4.3 & -3.3 \\
\hline $\mathrm{Pu}-238$ & -10.5 & -10.2 & Nd-143 & 0.5 & 2.1 \\
\hline Pu-239 & 4.9 & 9.1 & Nd-144 & -4.7 & -3.1 \\
\hline $\mathrm{Pu}-240$ & 1.5 & 1.8 & $\mathrm{Nd}-145$ & -4.4 & -3.1 \\
\hline $\mathrm{Pu}-241$ & -1.1 & 1.5 & Nd-146 & -1.5 & 0.3 \\
\hline $\mathrm{Pu}-242$ & -9.1 & -8.2 & Nd-148 & -1.5 & -0.1 \\
\hline $\mathrm{Np}-237$ & 2.7 & 3.1 & Nd-150 & 1.0 & 2.5 \\
\hline
\end{tabular}

Table 5: C/E -1 (\%) for Calvert Cliffs sample MKP109-P.

\section{Dancoff Factor Calculations}

SCALE utilizes Dancoff factors in MG resonance self-shielding calculations to account for interactions between fueled elements. SCALE analytically computes Dancoff factors for many uniform lattice structures. However, for strongly non-uniform lattice structures, such as boiling water reactor fuel assemblies with variable fuel rod enrichments and burnable absorbers plus varying moderator density regions, the use of a uniform lattice approximation may not produce accurate resonance self-shielded cross sections.

SCALE provides MCDancoff (Monte Carlo Dancoff) to calculate Dancoff factors for complicated, three-dimensional geometries, defined using SGGP. MCDancoff starts histories isotropically on the surface of the region for which the Dancoff factor is to be calculated and follows the path of each history scoring its encounters with regions of the same material in the system. A one-group cross-section library, which consists only of resonance integral cross-section data, is used to determine the total cross sections of the mixtures in the problem.

A current restriction of MCDancoff is that it can only calculate Dancoff factors for regions bounded by cylinders, spheres, or cuboids. Other simple bodies could be added in the future, but a general bounding surface would be impractical.

\section{Nuclear Data}

AMPX is a cross-section processing software package that has been developed and maintained at ORNL for more than 30 years and is completely independent of any other cross-section processing software package (Dunn and Greene, 2002). AMPX is used to process Evaluated Nuclear Data File (ENDF) nuclear data evaluations and provide CE, MG, and covariance data libraries for SCALE. The following summary identifies the recent AMPX updates that have led to improved nuclear data libraries for the SCALE code system for SCALE 6.2.

\subsection{Continuous-Energy Data}

Investigations into the CE data generated by the AMPX code system for deployment in SCALE 6.0 and 6.1 revealed a need for improvement in the $S(\alpha, \beta)$ treatment, especially for forward-peaked kinematics. For thermal moderators like hydrogen in $\mathrm{H}_{2} \mathrm{O}$, ENDF evaluations give detailed angular and exit energy information for incoherent elastic scattering. Information is given in the form of an $S(\alpha, \beta)$ function, where $\alpha$ is the momentum transfer and $\beta$ the energy transfer. AMPX uses the information from the ENDF/B evaluation to reconstruct the double differential scattering distribution. Prior to the development of the CE capabilities in SCALE, AMPX was primarily used to produce MG libraries, and AMPX collision kinematics distributions were provided as a function of cosine moment distributions instead of $\mathrm{CE}$ distributions. To support the $\mathrm{CE}$ capability development in SCALE, AMPX modules were developed to post-process the cosine moment distributions and recreate the original $\mathrm{CE}$ collision kinematics distributions. Once the $\mathrm{CE}$ distributions were reconstructed, the marginal and conditional probability distributions for exit angles and energies were generated (i.e., legacy CE kinematics distribution approach). For the CE libraries distributed with SCALE 6.0 and SCALE 6.1, AMPX used this legacy procedure to generate CE double-differential data from the cosine moment distributions. Following the release of SCALE 6.1, investigations revealed that this procedure introduced non-physical structure in the thermal scattering law data for water that resulted in a bias in some benchmark calculations. Based on the investigation, the AMPX collision kinematics processing procedures have been 
updated and modernized to produce $\mathrm{CE}$ probability distributions directly from the CE collision kinematics distributions, thereby eliminating the cosine moment distribution processing step. The SCALE ENDF/B-VII.0 data libraries have been regenerated using the new AMPX processing procedures, and the benchmark testing results with SCALE 6.2 show substantially improved results with the new CE data libraries. Select critical benchmark results for thermal mixed oxide (MOX) systems are provided in Figure 12: , which shows that the bias in the SCALE 6.2 CE results is reduced relative to SCALE 6.1. Additional details and further results are provided Marshall, et al, 2013.

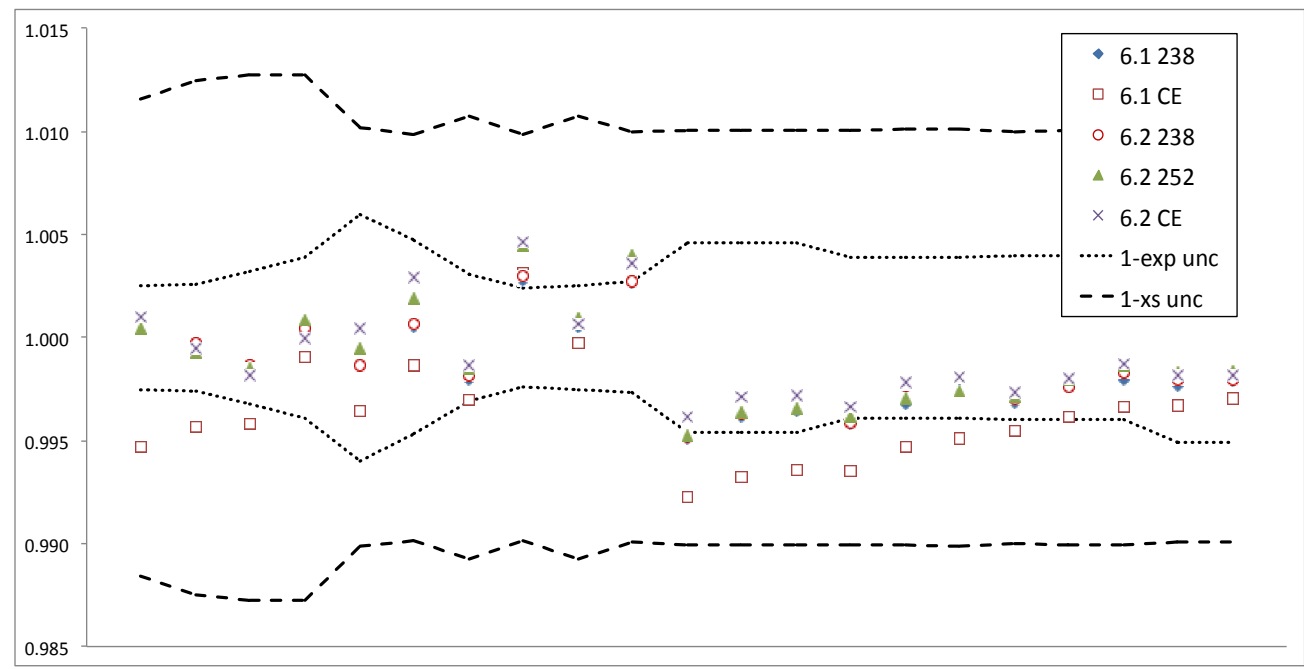

Figure 12: SCALE calculated results for IHECSBE thermal mixed oxide critical systems.

Historically, only neutron CE data libraries with a specific reaction subset of the ENDF libraries have been supported in KENO Monte Carlo criticality safety codes. To support CE depletion, sensitivity analysis, and coupled neutron-gamma shielding analysis, AMPX capabilities for the generation of CE neutron data have been improved and extended. Capabilities were added to generate gamma interaction data and to produce gamma yield data from neutron interactions. In addition, wide ranges of neutron interactions were also added in order to support creating reaction responses in Monaco.

Additionally, the probability tables that provide $\mathrm{CE}$ treatment in the unresolved resonance range have been improved, primarily through the inclusion of additional resolution and error correction. Testing with the new probability tables has shown reduced biases for systems that are sensitive to the intermediate energy range (Marshall, et al, 2013).

\subsection{Multigroup Data}

With regard to MG analyses, ORNL has performed detailed comparisons between SCALE CE and MG results, and a consistent bias has been observed between the 238-group and CE calculations. Subsequently, a detailed investigation was performed to develop a new group structure that would eliminate the consistent bias observed with the 238-group library, especially for light-water moderated systems. A new 252-group structure has been developed that provides a more detailed representation of the ${ }^{238} \mathrm{U}$ resonance structure, and AMPX has been used to develop a new 252-energy-group ENDF/B-VII.0 neutron cross-section library for SCALE 6.2. In addition to the new group structure, the 252-group library has been generated using a new weighting spectrum with improved resonance self-shielding parameters. Specifically, for actinides and hydrogen in $\mathrm{H}_{2} \mathrm{O}$, a temperature-dependent CE flux spectrum has been generated with the 1-D CE CENTRM module for a PWR pin cell, and the CE flux spectrum has been used to generate the 252-group library. Intermediate resonance parameters (lambdas) for all isotopes have been included in the library, which provides a capability for improved self-shielding with the Bondarenko method (Ellis, et al, 2012). Bondarenko shielding factors, as a function of background cross section and temperature, were computed for all actinides (except ${ }^{235} \mathrm{U},{ }^{238} \mathrm{U},{ }^{239} \mathrm{Pu}$, ${ }^{240} \mathrm{Pu}$, and ${ }^{241} \mathrm{Pu}$ ) using $\mathrm{CE}$ spectra calculated by CENTRM for homogeneous mixtures of the resonance material and hydrogen, corresponding to the respective background cross section. For the nuclides ${ }^{235} \mathrm{U},{ }^{238} \mathrm{U},{ }^{239} \mathrm{Pu}$, ${ }^{240} \mathrm{Pu}$, and ${ }^{241} \mathrm{Pu}$, the Bondarenko factors are based on CENTRM CE calculations for heterogeneous pin cell models that span the range of anticipated self-shielding conditions. The thermal cutoff in the 252-group library 
for thermal moderators and the free-gas approximation has been raised from $3 \mathrm{eV}$ to $5 \mathrm{eV}$. The new library has been tested by analyzing a wide variety of critical benchmark experiments and by comparing results with $\mathrm{CE}$ Monte Carlo results. The 252-group results presented in Figure 12: demonstrate consistent performance with the SCALE 6.2 CE results for the thermal MOX critical benchmark experiments. Based on additional studies with the 252-group library, computational benchmark comparisons with $\mathrm{CE}$ results at room temperature and elevated temperatures show agreement within $100 \mathrm{pcm}$ in most cases. In addition to the 252-group library, a new ENDF/B-VII.0 broad group library, with approximately 50-60 energy groups, is also under development for SCALE 6.2. The use of ENDF/B-VII.1 data with SCALE using the same techniques developed for the SCALE 6.2 ENDF/B-VII.0 libraries is also under investigation.

\section{Verification and Validation}

A recent focus of SCALE development has been improved verification and validation to minimize the number of bugs or errors, which are inconvenient to users and costly to patch. An increased focus on quality has led to orders-of-magnitude increases in routine testing throughout the design, implementation, and release of SCALE.

\subsection{Verification Suites}

After each incremental update to the source code, a suite of over 2000 test cases is run on a wide array of computer platform configurations, including Linux, Mac, and Windows with different compilers and compiler options. This rigorous testing is performed dozens of times each day, resulting in the quantification of performance with approximately 70,000 tests per day. The results of the tests and the associated changes are reported to an internal website, known as the SCALE Dashboard. All developers can review the Dashboard to monitor the performance of numerous SCALE features on different platforms with different compilers using a pass/fail metric without the need to configure and run all of these tests themselves.

Additional on-demand test suites quantify the performance of SCALE after significant code and/or nuclear data updates. For verification, a suite of over 6300 fixed-source tests was designed to quantify the transmission spectrum produced when a mono-energetic neutron or gamma source is targeted on a shield consisting of a specific material. For each test, the shield composition is varied to include each material available in the SCALE nuclear data library, and the source energy is varied incrementally across the full spectrum. A suite of over 5000 infinite medium criticality tests was developed to verify the performance of each nuclide at each available temperature with a thermal, intermediate, and fast spectrum. Another test suite examines neutron yield data with a Watt-spectrum neutron source placed at the center of a spherical shielding material. Outgoing particle fluxes are compared to reference values. This suite ensures that the basic particle transport data and methods are in compliance with the yield production data and methods. Additionally, a suite of several dozen reactor physics tests was designed to quantify performance with realistic conditions representing elevated temperatures, poisoned components, and depletion. The results of these thousands of tests are generated using each nuclear data library, inspected for reliability, and compared to the results produced by other codes in order to identify and resolve any discrepancies.

\subsection{Validation Suites}

The SCALE Verified, Archived Library of Inputs and Data (VALID) validation suite includes over 400 nuclear criticality experiments, spanning a broad range of fuels, moderators, reflectors, and poisons, based on critical experiments collected from the IHECSBE. Also included are several shielding benchmarks, which consist of

${ }^{252} \mathrm{Cf}$ neutron transmission through an iron sphere (Sajo, et al, 1993). The experiments are routinely examined to quantify the performance of SCALE simulations relative to measured data.

\section{User Interfaces}

SCALE includes a number of graphical user interfaces to provide convenient means of generating input, executing SCALE, and visualizing models and data. GeeWiz (Graphically Enhanced Editing Wizard), shown in Figure 13, is a Windows user interface that provides a control center for setup, execution, and viewing results for most of SCALE's computational sequences. GeeWiz is coupled with the KENO3D interactive visualization program for Windows for solid-body rendering of KENO geometry models, as demonstrated in Figure 1. The Javapeño (Java Plots Especially Nice Output) multiplatform interface provides 2D and 3D plotting of cross-section and cross-section-covariance data, multigroup fluxes and reaction rates from KENO, and sensitivity data from TSUNAMI (shown in Figure 10). The MeshView multiplatform interface produces 2D contour views of mesh data and mesh results from Monaco and KENO (examples in Figures 7-9), and ChartPlot 
provides for energy-dependent plots of Monaco results. Additionally, several codes provide HTML-formatted output, in addition to the standard text output, to provide convenient navigation through the computed results using most common web browsers with interactive color-coded output and integrated data visualization tools, as shown in Figure 14.

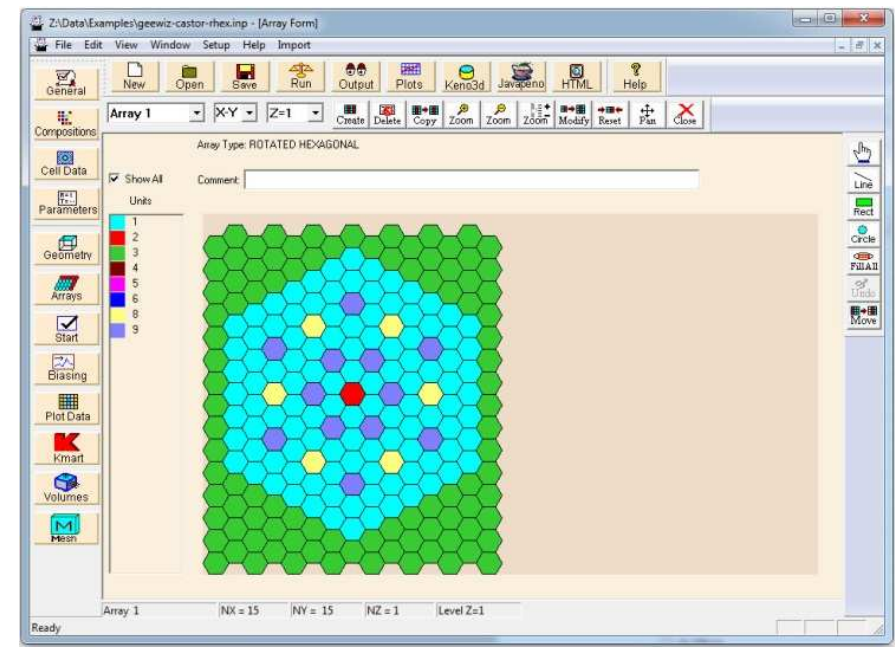

Figure 13: Simplified array data entry with GeeWiz.

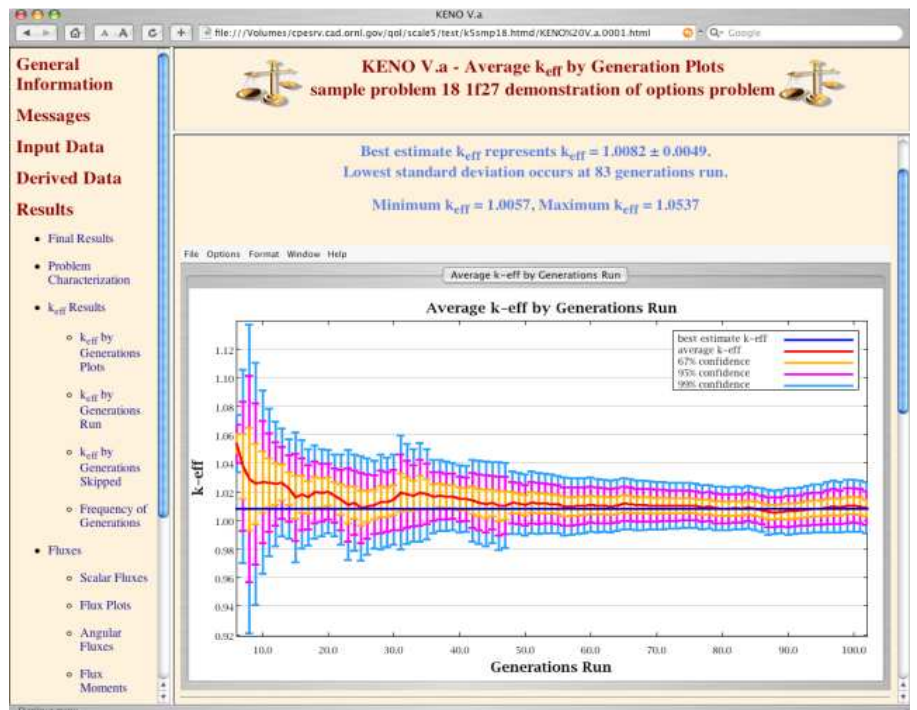

Figure 14: HTML-formatted output from KENO with embedded Javapeño applet.

\section{Distribution}

\subsection{Quality Assurance}

The SCALE QA program has been updated in 2013 to provide an improved means of providing high-quality software and data for the user community. A new QA program has been implemented, which is compliant with ISO 9001 (ANSI, 2008), DOE 414.1D (DOE, 2011) the ORNL Standards-Based Management System, and is consistent with ASME NQA-1 (ASME, 1994).

\subsection{Availability}

The distribution of SCALE to end users is subject to US export control regulations, and each user must be individually licensed through an authorized distribution center. SCALE licenses are primarily issued through the Radiation Safety Information Computational Center (RSICC) at ORNL with mirrors at the Organization for Economic Cooperation and Development (OECD) Nuclear Energy Agency (NEA) Data Bank in France and the Research Organization for Information Science and Technology (RIST) in Japan. 


\subsection{User Base}

From 2004-2014, over 8000 licenses of SCALE 5.0-6.1 have been issued to 6000 individuals in 52 nations.

\subsection{Technical Support}

Support for SCALE users is provided through the availability of 10 weeks of public training courses each year, workshops provided at conferences, an e-mail helpline, an online discussion forum, and a bi-annual newsletter.

\section{Conclusion}

SCALE provides numerous verified and validated production Monte Carlo capabilities for criticality safety, radiation shielding, sensitivity analysis, and depletion. Numerous enhancements in SCALE 6.2 will provide enhanced capabilities, especially for $\mathrm{CE}$ calculations with reduced computational requirements and improved solution fidelity.

\section{Acknowledgments}

SCALE is sponsored by the US Nuclear Regulatory Commission and the US Department of Energy.

This manuscript has been authored by the Oak Ridge National Laboratory, managed by UT-Battelle LLC under Contract No. DE-AC05-00OR22725 with the US Department of Energy. The US Government retains and the publisher, by accepting the article for publication, acknowledges that the US Government retains a nonexclusive, paid-up, irrevocable, worldwide license to publish or reproduce the published form of this manuscript, or allow others to do so, for US Government purposes.

\section{References}

ANSI/ISO/ASQ Q9001-2008, Quality Management Systems.

ASME NQA-1-1994, Quality Assurance Requirements for Nuclear Facility Applications.

Becker, B. et al., 2009. Proof and Implementation of the Stochastic Formula for Ideal Gas, Energy Dependent Scattering Kernel. Ann. Nucl. Energy, 36, 470-474.

Blomquist, R. N., et al., 2006. Source Convergence in Criticality Safety Analysis: Phase I: Results for Four Test Problems, Organization for Economic Cooperation and Development, Nuclear Energy Agency, NEA No. 5431, ISNB 92-64-02304-6.

DeHart, M. D., Bowman, S. M., 2011. Reactor Physics Methods and Analysis Capabilities in SCALE. Nucl. Technol., 174, 196-213.

DOE Order 414.1D, Quality Assurance, 2011.

Dunn, M. E., Greene, N. M., 2002. AMPX-2000: A Cross-Section Processing System for Generating Nuclear Data for Criticality Safety Application. Trans. Am. Nucl. Soc., 86, 118-199.

Ellis, R. J. et al., 2012. Generation of a Broad-Group HTGR Library for Use with SCALE, NUREG/CR-7106 (ORNL/TM-2011/298), prepared for the US Nuclear Regulatory Commission by Oak Ridge National Laboratory, Oak Ridge, TN, June 2012.

Evans, T. M. et al., 2010. Denovo: A New Three-Dimensional Parallel Discrete Ordinates Code in SCALE. Nucl. Technol., 171, 171-200.

Gabriel, E. et al., 2004. Open MPI: Goals, Concept, and Design of a Next Generation MPI Implementation. In Proceedings, 11th European PVM/MPI Users' Group Meeting, 97-104, Budapest, Hungary.

Goluoglu, S. et al., 2012. SCALE Continuous-Energy Monte Carlo Depletion with Parallel KENO in TRITON. Trans. Am. Nucl. Soc., 106, 723-725.

Goluoglu, S. et al., 2011. Monte Carlo Criticality Methods and Analysis Capabilities in SCALE. Nucl. Technol., 174, 214-235.

Hart, S., 2013. Implementation of the Doppler Broadening Rejection Correction in KENO. Trans. Am. Nucl. Soc., 108, 423-426.

Hoogenboom, J. E. et al., 2011. Monte Carlo Performance Benchmark for Detailed Power Density Calculation 
in a Full Size Reactor Core. http://www.oecd-nea.org/dbprog/MonteCarloPerformanceBenchmark.htm,

Ibrahim, A. M. et al., 2013. Hybrid Technique in SCALE for Fission Source Convergence Applied to Used Nuclear Fuel Analysis. In preparation for the 2013 Topical Meeting on Nuclear Criticality Safety (NCSD 2013), Wilmington, NC, September 29-October 3, 2013.

Ibrahim, A. M. et al., 2012. Acceleration of Monte Carlo Criticality Calculations Using Deterministic-Based Starting Sources. PHYSOR 2012, Knoxville, TN, April 15-20, 2012.

Ibrahim, A. M. et al., 2011. Acceleration of Monte Carlo Criticality Calculations Using Deterministic-Based Starting Sources. Trans. Am. Nucl. Soc., 105, 539-541.

Ilas, G. et al., 2010. Analysis of Experimental Data for High Burnup PWR Spent Fuel Isotopic Validation-Calvert Cliffs, Takahama, and Three Mile Island Reactors. NUREG/CR-6968 (ORNL/TM-2008/071), prepared for the US Nuclear Regulatory Commission by Oak Ridge National Laboratory, Oak Ridge, TN.

International Handbook of Evaluated Criticality Safety Benchmark Experiments NEA/NSC/DOC(95)03, 2012. Organization for Economic Co-operation and Development-Nuclear Energy Agency (OECD-NEA).

Larson, N., 2008. Updated User's Guide for Sammy: Multilevel R-Matrix Fits to Neutron Data Using Bayes' Equations, ORNL/TM-9179/R8, Oak Ridge National Laboratory, Oak Ridge, TN.

Marshall, W. J. et al., 2013. Validation of Criticality Safety Calculations with SCALE 6.2. Proceedings of ANS NCSD 2013 - Criticality Safety in the Modern Era: Raising the Bar, Wilmington, NC, September 29-October 3, 2013, on CD-ROM, American Nuclear Society, LaGrange Park, IL.

Oak Ridge National Laboratory, 2011. SCALE: A Comprehensive Modeling and Simulation Suite for Nuclear Safety Analysis and Design, ORNL/TM-2005/39, Version 6.1, Oak Ridge, TN. Available from Radiation Safety Information Computational Center at Oak Ridge National Laboratory as CCC-785.

Peplow, D. E., 2011. Monte Carlo Shielding Analysis Capabilities with MAVRIC. Nucl. Technol., 174, 289-313.

Perfetti, C. M., Rearden, B. T., 2013. Development of a SCALE Tool for Continuous-Energy Eigenvalue Sensitivity Coefficient Calculations. Proceedings of Joint International Conference on Supercomputing in Nuclear Applications and Monte Carlo 2013 (SNA + MC 2013) La Cité des Sciences et de l'Industrie, Paris, France, October 27-31, 2013.

Rearden, B. T. et al., 2013. Quantification of Uncertainties and Correlations in Criticality Experiments. Proceedings of ANS NCSD 2013 - Criticality Safety in the Modern Era: Raising the Bar, Wilmington, NC, September 29-October 3, 2013, on CD-ROM, American Nuclear Society, LaGrange Park, IL.

Rearden, B. T. et al., 2011. Sensitivity and Uncertainty Analysis Capabilities and Data in SCALE. Nucl. Technol., 174, 236-288.

Rearden, B. T., 2004. Perturbation Theory Eigenvalue Sensitivity Analysis with Monte Carlo Techniques. Nucl. Sci. Eng., 146, 367-382.

Sajo, E. et al., 1993. Comparison of Measured and Calculated Neutron Transmission Through Steel for a ${ }^{252} \mathrm{Cf}$ Source. Ann. Nucl. Energy, 20, 585-604.

Ueki, T., Brown, F. B., 2005. Stationarity Modeling and Informatics-Based Diagnostics in Monte Carlo Criticality Calculations. Nucl. Sci. Eng., 149, 38-50.

Ueki, T., et al., 1997. Error Estimations and Their Biases in Monte Carlo Eigenvalue Calculations. Nucl. Sci. Eng., 125, 1.

Williams, M. L. et al., 2013. SCALE Uncertainty Quantification Methodology for Criticality Safety Analysis of Used Nuclear Fuel. Proceedings of ANS NCSD 2013 - Criticality Safety in the Modern Era: Raising the Bar, Wilmington, NC, September 29-October 3, 2013, on CD-ROM, American Nuclear Society, LaGrange Park, IL.

Williams, M. L., 2011. Resonance Self-Shielding Methodologies in SCALE 6. Nucl. Technol., 174, 149-168.

Williams, M. L. et al., 2001. Eigenvalue Sensitivity Theory for Resonance-Shielded Cross Sections. Nucl. Sci. Eng., 138, 177-191. 